\title{
Systemic Galectin-3 in Smokers with Chronic Obstructive Pulmonary Disease and Chronic Bronchitis: The Impact of Exacerbations
}

This article was published in the following Dove Press journal: International Journal of Chronic Obstructive Pulmonary Disease

\author{
Martina Sundqvist $\mathbb{D}^{1, *}$ \\ Kristina Andelid (D) ${ }^{2, *}$ \\ Ann Ekberg-Jansson ${ }^{3}$ \\ Johan Bylund (iD) 4 \\ Anna Karlsson-Bengtsson (iD) ${ }^{1,5}$ \\ Anders Lindén (iD) ${ }^{6,7}$ \\ 'Department of Rheumatology and \\ Inflammation Research, Institute of \\ Medicine, Sahlgrenska Academy, University \\ of Gothenburg, Gothenburg, Sweden; \\ ${ }^{2}$ COPD Center, Department of Internal \\ Medicine and Clinical Nutrition, Institute of \\ Medicine, Sahlgrenska Academy, University \\ of Gothenburg, Gothenburg, Sweden; \\ ${ }^{3}$ Department of Respiratory Medicine and \\ Allergology, Institute of Medicine, \\ Sahlgrenska Academy, University of \\ Gothenburg, Gothenburg, Sweden; \\ ${ }^{4}$ Department of Oral Microbiology and \\ Immunology, Institute of Odontology, \\ Sahlgrenska Academy, University of \\ Gothenburg, Gothenburg, Sweden; \\ ${ }^{5}$ Department of Biology and Biological \\ Engineering, Chalmers University of \\ Technology, Gothenburg, Sweden; ${ }^{6}$ Unit for \\ Lung and Airway Research, Institute of \\ Environmental Medicine, Karolinska \\ Institutet, Stockholm, Sweden; ${ }^{7}$ Karolinska \\ Severe COPD Center, Department of \\ Respiratory Medicine and Allergy, Karolinska \\ University Hospital, Stockholm, Sweden \\ *These authors contributed equally to this \\ work
}

Purpose: The carbohydrate-binding protein Galectin-3 is increased in several inflammatory diseases and has recently been forwarded as a systemic biomarker in chronic obstructive pulmonary disease (COPD). In this longitudinal study, we characterized the level of systemic Galectin-3 using blood from smokers with a history of COPD and chronic bronchitis (COPD-CB), during stable clinical conditions and exacerbations.

Patients and Methods: The study population comprised 56 long-term smokers with COPD-CB, 10 long-term smokers without lung disease (LTS) and 10 clinically healthy never-smokers (HNS). Blood samples were analyzed for levels of Galectin-3, leukocyte populations and C-reactive protein (CRP). In addition, sputum samples from the COPD$\mathrm{CB}$ group were analyzed for bacterial growth.

Results: When comparing stable clinical conditions and exacerbations in the COPD-CB group, we found that the level of Galectin-3, just like that of CRP, leukocytes and neutrophils, respectively, was increased during exacerbations. However, this exacerbation-associated increase of Galectin-3 was modest. During stable clinical conditions of COPD-CB, the level of Galectin-3 was not elevated in comparison with HNS or LTS. Nor did this level of Galectin3 distinguish patients that remained in a clinically stable condition throughout the study to those that developed an exacerbation. In addition, neither during stable clinical conditions nor during exacerbations, did the presence of bacterial growth in sputum alter Galectin-3 levels. In contrast to Galectin-3, the level of CRP, leukocytes and neutrophils, respectively, were increased during clinical stable conditions in the COPD-CB group compared with the other groups and were further enhanced during exacerbations.

Conclusion: Systemic Galectin-3 is increased in a reproducible but modest manner during exacerbations in smokers with COPD-CB. During stable clinical conditions, the level of systemic Galectin-3 does not distinguish patients that remain clinically stable from those that develop exacerbations. This makes it less likely that systemic Galectin-3 may become a clinically useful biomarker in the current setting.

Keywords: COPD, CRP, airflow limitation, exacerbation
Correspondence: Martina Sundqvist Department of Rheumatology and Inflammation Research, Institute of Medicine, Sahlgrenska Academy, University of Gothenburg, Guldhedsgatan I0A, Gothenburg, S-413 46, Sweden Email martina.sundqvist@rheuma.gu.se

\section{Introduction}

Chronic obstructive pulmonary disease (COPD) is a burden to public health and a global killer, projected to soon become the third leading cause of disease-related death worldwide. ${ }^{1}$ According to the Global initiative for Chronic Obstructive Lung Disease (GOLD), COPD is characterized by persistent airflow limitation associated with a chronic inflammatory response that is caused by long-term exposure to noxious gases and/or toxic 
particles. ${ }^{2}$ The persistent airflow limitation is primarily caused by bronchiolitis that causes irreversible obstruction but it may be enhanced by parenchymal destruction (emphysema) and excessive mucus production (chronic bronchitis). Many patients with COPD suffer from repeated episodes of severely increased symptoms (exacerbations), characterized by even more pronounced local and systemic inflammation, leading to the transient deterioration of lung function, reduced quality of life, hospitalization, and risk for further disease progression. $^{2}$ In the Western world, the most common cause of COPD is smoke from tobacco but smoke from other types of biomass may also increase the risk for COPD. ${ }^{3-5}$

From a clinical point-of-view, it seems critical to prevent exacerbations of $\mathrm{COPD}$, in order to preserve lung function, for the sake of the patient and for the sake of the health economy. ${ }^{3}$ However, despite substantial research efforts to identify biomarkers in blood that can predict exacerbations, ${ }^{2,6-13}$ none have so far proven practically useful in the clinical setting for the general assessment of exacerbations in individual patients.

Galectin-3 (Mac-2) is a $\beta$-galactoside-binding protein produced by various tissues and cell types in humans, ${ }^{14-16}$ including monocytes and, even more so, macrophages. ${ }^{17}$ This is noteworthy given that the number of alveolar macrophages is enhanced in COPD. ${ }^{18,19}$ Interestingly, Galectin-3 has been implied as a key regulator of innate immunity, ${ }^{20}$ due to its ability to enhance phagocytosis, ${ }^{21-23}$ initiate the production of reactive oxygen species ${ }^{24,25}$ and promote cell adhesion. ${ }^{26,27}$ With reference to clinical pathology, previous studies have demonstrated enhanced systemic levels of Galectin-3 in patients with various types of cancer ${ }^{28-30}$ and heart failure, ${ }^{31,32}$ respectively. In terms of airway disease, one study has demonstrated an increased level of Galectin-3 locally in the airways during stable clinical conditions for very severe COPD and chronic bronchitis (CB), with all investigated patients being former smokers. ${ }^{33}$ Another study demonstrated that the systemic level of Galectin-3 in the blood is increased during exacerbations in patients with moderate to severe COPD. ${ }^{34}$ However, the described enhancement of Galectin-3 emerged as modest, possibly due to different confounders like the variation in pharmacotherapy and the exact timing of sampling. Additional confounders were evident as well, since the patients with COPD comprised both never-, ex- and current-smokers and it was unclear whether they suffered from CB. ${ }^{34}$ The latter might be important since $\mathrm{CB}$ is a comorbidity that increases the risk for exacerbations. ${ }^{35}$

In the present study, we assessed systemic levels of Galectin- 3 by sampling blood before and during exacerbations in patients with a confirmed history of COPD and CB (COPD$\mathrm{CB})$. We compared these systemic levels of Galectin-3 to those in matching samples collected from clinically healthy never-smokers (HNS) and to those in samples from long-term smokers (LTS) with normal lung function/spirometry and without COPD-CB. Notably, we confirmed a consistent status of current smoking among all included LTS and COPD-CB patients, by assessing the nicotine metabolite cotinine. Finally, we related Galectin-3 in the blood to the archetype marker of systemic inflammation in the blood, C-reactive protein (CRP), and to the detection of local bacterial pathogens in airway samples from the COPD-CB patients.

\section{Patients and Methods}

\section{Study Population}

The study subjects were recruited by advertisement in the newspaper or from the outpatient clinic at the Department of Respiratory Medicine at Sahlgrenska Hospital in Gothenburg, Sweden. The main study group included 56 long-term smokers ( $>10$ pack-years) who were all current smokers (positive for cotinine) with a confirmed COPD diagnosis (Table 1). Among these 56 patients, 53 patients (95\%) had a COPD diagnosis in their patient records prior to screening and three received the diagnosis during screening. ${ }^{36,37}$ For all these patients, the diagnosis of COPD was set by experienced clinicians. ${ }^{2}$ Thus, all included patients displayed spirometry results with an $\mathrm{FEV}_{1} / \mathrm{FVC}$ ratio (\%) of less than $70 \%$ and GOLD-staging was performed based upon $\mathrm{FEV}_{1}(\%$ predicted). ${ }^{38}$ The diagnosis "chronic bronchitis" was set after confirmation that the patient had a productive cough during at least 3 months per year for two subsequent years, in accordance with the classic criteria by Kim et al. ${ }^{39}$

During the inclusion visit, we ascertained that all patients had been without clinical signs of infections during the four most recent weeks prior to inclusion. Other than CB, 43 out of the 56 patients (77\%) suffered from comorbidities including osteoporosis, hypertension, diabetes and depression (Table 2), which are common amongst patients with COPD. ${ }^{40}$ The exclusion criteria in this COPD-CB group were as follows: i) long-term oxygen therapy, ii) regular use of oral glucocorticoids, iii) any additional lung disease other than COPD or CB (eg, pulmonary fibrosis, asbestosis, alpha-1 antitrypsin deficiency or sarcoidosis), iv) allergy, sensitization or asthma or v) other serious diseases (including congestive heart failure and ischemic heart disease, mental illness [apart from depression], malignancies and chronic inflammatory diseases such as rheumatoid arthritis and inflammatory bowel disease). 
Table I Clinical Characteristics of Study Subjects at Inclusion ${ }^{\mathrm{a}}$

\begin{tabular}{|c|c|c|c|}
\hline & HNS & LTS & COPD-CB \\
\hline $\mathrm{n}$ & 10 & 10 & 56 \\
\hline Sex (Male/Female) & $3 / 7$ & $2 / 8$ & $23 / 33$ \\
\hline Age (years) & $68(47-70)$ & $50(26-64)^{*(t, \S)}$ & $63(45-76)$ \\
\hline Pack-years (range) & $0(0)$ & $28(13-43)$ & $40(14-156)$ \\
\hline BMI $\left(\mathrm{kg} / \mathrm{m}^{2}\right)$ & $25(20-31)$ & $26(22-31)$ & $26(17-34)$ \\
\hline FEV (\% of predicted) & $120(97-137)$ & $106(83-119)$ & $60(29-97)^{*(+, \neq)}$ \\
\hline $\mathrm{FEV}_{\mathrm{I}} / \mathrm{FVC}(\mathrm{FEV} \%)$ & $79(73-84)$ & 77 (73-84) & $55(28-68)^{*(+, f)}$ \\
\hline DLCO (\% of predicted) & $98(75-139)$ & $87(77-111)$ & $70(44-110)^{*(t, \neq)}$ \\
\hline CRP (mg/L) & $0.8(0.2-5.7)$ & $0.7(0.2-5.8)$ & $1.7(0.3-15.0)^{*(+)}$ \\
\hline Leukocytes $\left(\times 10^{9} / \mathrm{L}\right)$ & $5.0(4.0-7.6)$ & $7.5(4.2-12.5)$ & $7.5(3.9-14.6)^{*(+)}$ \\
\hline \multicolumn{4}{|l|}{ Medications ( $(n)^{b}$} \\
\hline Untreated & - & - & 4 \\
\hline Bronchodilator & - & - & 44 \\
\hline Glucocorticoid & - & - & 8 \\
\hline Combination & - & - & 23 \\
\hline Mucolytics & - & - & 18 \\
\hline
\end{tabular}

Notes: Data is given as median (range minimum-maximum value) based on the total $n$ in each group (HNS, $n=10 ; L T S, n=10 ; C O P D-C B, n=56)$ except for: pack-years, include nine LTS individuals; DLCO, include 54 COPD-CB patients; CRP, include 55 COPD-CB patients.

Statistical analysis: Mann-Whitney $U$ test showed no statistical significant difference in pack-years between LTS and COPD-CB patients $(p=0.057)$; Kruskal-Wallis test followed by Dunn's multiple comparison test revealed statistical significant lower age in LTS compared to both HNS $\left({ }^{\dagger}\right)$ and COPD-CB patients $\left({ }^{\S}\right)$, statistical significant lower $\mathrm{FEV}_{1}, \mathrm{FEV} / \mathrm{FVC}$ and DLCO values in the COPD-CB patients compared to both HNS and LTS $\left({ }^{\ddagger}\right)$ and statistical significant higher CRP and leukocytes levels in the COPD-CB patients compared HNS $\left({ }^{\dagger}\right)$. No statistical significant differences were found for BMI. Statistically significant differences are indicated as follows: $(*) p$-value $<0.05$. ${ }^{a}$ Some parameters have been described in our previous publications. ${ }^{36,37}$ b All medications were administered by inhalation, except mucolytics ( $\mathrm{N}$-acetylcysteine, oral intake). Bronchodilator include short- and long-acting anticholinergics and $\beta 2$-agonists and combination include inhalers with a bronchodilator and a glucocorticoid.

Abbreviations: BMI, body mass index; CRP, C-reactive protein; DLCO, gas diffusion capacity for carbon monoxide; FEV forced vital capacity; HNS, healthy never smokers; LTS, long-term smokers without chronic obstructive pulmonary disease and chronic bronchitis (COPD-CB).

Two control groups were also recruited as follows: ten clinically healthy never-smokers (HNS; negative for cotinine) and ten long-term smokers (LTS) who were all current smokers (positive cotinine) but had normal lung function/spirometry. The latter group had no chronic bronchitis or other lung diseases, as judged from their history. The clinical characteristics of all study subjects/patients are summarized in Table 1. Of note, the original study population has been described in two previous publications, focusing on primary outcomes other than Galectin3. ${ }^{36,37}$ Thus, beyond Galectin-3, certain clinical and laboratory data from the included study subjects have previously been utilized in a different scientific context.

\section{Study Protocol}

Briefly, before inclusion, all subjects were examined during stable clinical conditions and critically evaluated regarding the results of physical examination, lung function tests (spirometry, gas diffusion capacity), chest X-ray, and sputum, urine and blood samples, as previously described. ${ }^{37}$ Both blood and sputum samples (study material) were collected from the patients at the time of inclusion and blood samples were thereafter collected every
15 th week during the following 60 weeks. If the patient experienced signs of an exacerbation, an extra visit was scheduled for the collection of blood and sputum samples, with the aim to obtain samples prior to any emergency medication was given because of the exacerbation.

\section{Bacteria in Sputum Samples}

Fresh sputum samples were collected from the COPD-CB group at the time of inclusion and during exacerbations. To assess whether the sputum samples were representative for the lower airways, all samples were evaluated for cell morphology before utilized for the culture of bacteria as previously described. ${ }^{36,37}$

\section{Cotinine}

Fresh urine samples collected at the time of inclusion were analyzed for cotinine, the major metabolite of nicotine. This analysis was performed using a visual one-step immunoassay for the qualitative detection of cotinine (One-Step Cotinine Test $^{\mathrm{TM}}$ \#008A086, Ulti Med Products, Ahrensburg, Germany), with a cut-off concentration of $200 \mathrm{ng} / \mathrm{mL}$, according to manufacturer's instructions. 
Table 2 Exacerbation Frequency and Comorbidities

\begin{tabular}{|l|l|l|}
\hline Disease Severity $^{\text {a }}$ & Mild & Severe \\
\hline $\mathrm{n}$ & 39 & 17 \\
\hline Exacerbations prior study (n) $^{\mathrm{b}}$ & & \\
$\quad$ Do not recall & 10 & 3 \\
0 & 5 & 3 \\
$\mathrm{I}-2$ & 19 & 7 \\
$\geq 3$ & 5 & 4 \\
\hline Exacerbations during study (n) & & \\
0 & 16 & 6 \\
I & 13 & 6 \\
2 & 7 & 2 \\
3 & 3 & 3 \\
\hline Comorbidities (n) & & \\
None & 8 & 5 \\
Hypertension & 8 & 3 \\
Cardiac disease & & 1 \\
Osteoporosis/Osteopenia & 3 & 1 \\
Hypercholesterolemia & 7 & 3 \\
Type 2 diabetes mellitus & 8 & - \\
Hypothyroidism & 3 & 5 \\
Sleep apnea & 5 & - \\
Depression/anxiety & 14 & \\
\hline
\end{tabular}

Notes: ${ }^{\text {aT }}$ The COPD-CB patients were classified according to the Global initiative for Chronic Obstructive Lung Disease (GOLD) criteria as of $2011^{38}$; mild, COPD$\mathrm{CB}$ corresponding to GOLD stage I and II; severe, COPD-CB corresponding to GOLD stage III and IV. 'Based on the year prior study inclusion and estimated by a questionnaire consisting of five domains: number of exacerbations: $0 ; 1-2 ; 3 ;>3$ or, do not recall/unsure. ${ }^{c}$ Cardiac disease includes atrial flutter, mechanical valve surgery, silent myocardial infarction and sinus tachycardia.

\section{Galectin-3}

Fresh blood samples were processed to obtain serum and were then frozen $\left(-80^{\circ} \mathrm{C}\right)$. After thawing, Galectin-3 protein was quantified utilizing a commercial enzyme-linked immunosorbent assay (ELISA) on a microtiter platform with a measurement range of 1.4-94.8 $\mathrm{ng} / \mathrm{mL}$ (BGM Galectin-3 Assay \#12836, BG Medicine, Inc. Waltham, MA, USA), in accordance with the manufacturer's instructions.

\section{C-Reactive Protein (CRP)}

The level of CRP was assessed in serum samples utilizing a particle-enhanced immunoturbidimetric assay in which human CRP agglutinate with latex particles coated with anti-CRP antibodies (Tina-quant C-reactive protein highsensitive assay ${ }^{\mathrm{TM}}$ HS No 1972944001; Hoffmann-La Roche Ltd, Basel, Switzerland) at the Department of Clinical Chemistry, Sahlgrenska University Hospital, Gothenburg, Sweden, in accordance with the manufacturer's instructions.

\section{Leukocytes}

The level (absolute concentration) of all (total) leukocytes and the respective subpopulation was quantified by hematology analysis (ADVIA 2120i Hematology system ${ }^{\mathrm{TM}}$; Siemens, Duisburg, Germany) at the Department of Clinical Chemistry, Sahlgrenska University Hospital, Gothenburg, Sweden, in accordance with the manufacturer's instructions.

\section{Statistical Analysis}

Non-parametric statistical analyses were performed in Graph Pad Prism version 8.3.1 (GraphPad Software, San Diego, CA, USA) using a Mann-Whitney $U$ test, Wilcoxon signed-rank test or Kruskal-Wallis test followed by Dunn's multiple to evaluate differences between groups or Spearman's rank correlation to evaluate correlations between Galectin-3 and CRP or leukocytes during exacerbations. The specific statistical tests applied are presented in the respective figure legend and table footnotes. A $p$-value of $<0.05$ was regarded as statistically significant and the $p$-value was indicated as follows: $\left.{ }^{*}\right) p<0.05 ;{ }^{*}$ ) $p<0.01 ; * * *) p<0.001$ and $* * * *) p<0.0001$.

\section{Results}

\section{Characteristics of the Study Population}

The clinical characteristics of the COPD-CB group and control groups (HNS and LTS) are described in Table 1. The spirometric parameters measured $\left(\mathrm{FEV}_{1}, \mathrm{FEV}_{1} / \mathrm{FVC}\right.$ and DLCO) were all altered in a statistically significant and typical manner in the COPD-CB group as compared to both control groups. However, the historic tobacco load (number of pack-years) did not markedly differ between the COPD-CB and LTS group. In $52(93 \%)$ of the patients in the COPD-CB group, inhalation (bronchodilators and/or glucocorticoids) and/or orally administered mucolytics were utilized by the patients during stable clinical conditions (Table 1). The patients in the COPD-CB group displayed severity corresponding to GOLD stage I, II, III and IV. ${ }^{38}$ As assessed by a questionnaire, 43 (77\%) of the patients in the COPD-CB group recalled having exacerbations the last year preceding inclusion in the study. Throughout the study, 34 (61\%) of the 56 patients in the COPD-CB group suffered from exacerbations (Table 2).

\section{Galectin-3 During Stable Clinical Conditions}

During the stable clinical conditions at the time of inclusion, the (median) level of Galectin-3 in serum was similar 
for the COPD-CB and the HNS and LTS control group, respectively (Figure 1A).

We also evaluated whether the level of Galectin-3 during stable clinical conditions (at the time of inclusion) predicts an upcoming exacerbation in the COPD-CB group. We did this by comparing the level for the 22 (39\%) patients that remained in a clinically stable condition throughout the study, with that of the $34(61 \%)$ that developed an exacerbation during the study. However, we detected no substantial difference in the level of Galectin-3 during stable clinical conditions for these two sub-groups (Figure 1B). In addition, we analyzed if the level of Galectin-3 during stable clinical conditions (at the time of inclusion) correlated with the number of exacerbations in the patients from the COPD-CB group that experienced this condition during the study. However, there was no statistically significant correlation in this respect (Spearman's Rho $=-0.15,95 \%$ confidence interval -0 .40-0.13, number of exacerbations are shown in Table 2).

For comparison to Galectin-3, we also determined the level of the acute phase reactant CRP. ${ }^{5,41-43}$ Here, the level of CRP was increased in the COPD-CB group at the time of inclusion, as compared to the HNS and LTS control group, respectively. However, just like the case for Galectin-3, the level of CRP was not markedly different for the COPD-CB patients with and without exacerbations, respectively (Figure 1C).

\section{Galectin-3 During Exacerbations}

Galectin-3 in serum was also quantified in 29 (85\%) of the 34 patients in the COPD-CB group that suffered from exacerbations during the 15-month course of the study. For all these 29 patients, blood and sputum samples were collected prior to the administration of emergency medication. By comparing the level of Galectin-3 at the last regular visit prior to the exacerbation (during stable clinical conditions) with that obtained during the exacerbation, a statistically significant increase in the level of Galectin-3 was detected (Figure 2A). Here, the median (range) time from a prior visit to exacerbation was 12.5 (2.7-15.5) weeks. Similar to Galectin-3, CRP displayed an increased level during an exacerbation, as compared to the most recent regular visit prior to the exacerbation (Figure 2B).

\section{Galectin-3 in Relation to Bacteria in Sputum Samples}

We also evaluated whether the level of Galetin-3 in the COPD-CB group was different for the patients with the growth of oropharyngeal flora, common respiratory pathogens or opportunistic pathogens. However, we detected no reproducible difference for these sub-groups, neither during stable clinical conditions (at the time of inclusion) nor during exacerbations (Figure $3 \mathrm{~A}$ and $\mathrm{B}$ ).

\section{Leukocytes During Exacerbations}

We compared the levels (absolute concentration) of total leukocytes, as well as the subpopulations, monocytes and neutrophils, in blood from the COPD-CB group in samples obtained at the most recent visit prior to the exacerbation (during stable clinical conditions) to the one obtained during exacerbations. Here, we detected a statistically significant increase in the level of total leukocytes and neutrophils during exacerbations (Figure 4A and B). For monocytes, there was a trend towards a similar increase (Figure 4C).
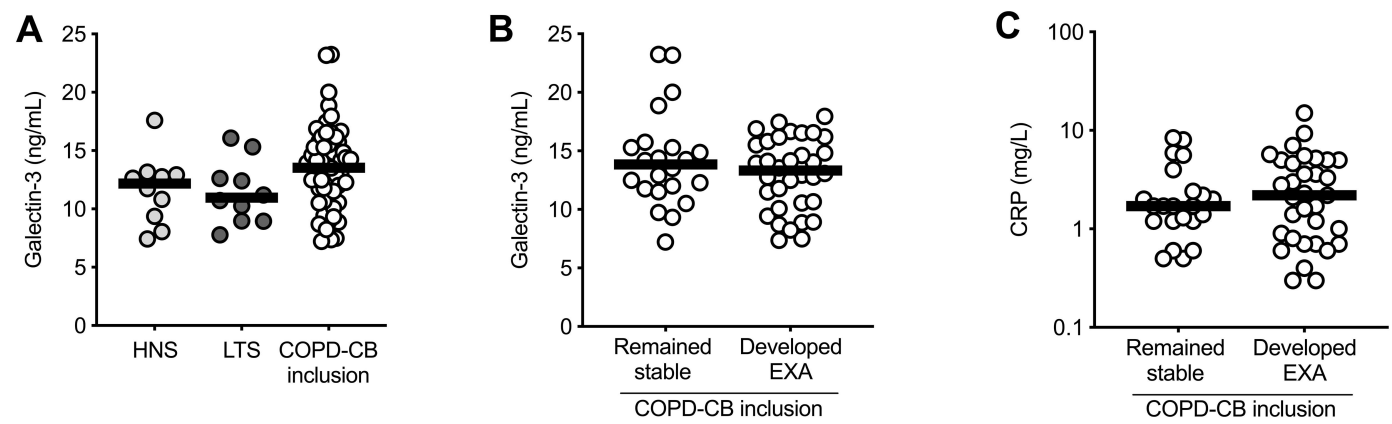

Figure I Serum levels of Galectin-3 during stable clinical conditions. (A) The level of Galectin-3 in serum from healthy never smoker (HNS; $\mathrm{n}=10$, light grey dots) as well as from long-term smokers without (LTS; $n=10$, dark grey dots) and with chronic obstructive pulmonary disease and chronic bronchitis during stable clinical conditions at the time inclusion (COPD-CB inclusion; $n=56$, white dots). (B) The level of Galectin-3 and (C) the level of CRP in serum from COPD-CB patients at the time of inclusion that either remained in a stable clinical phase (remained stable; $n=22$ for both Galectin-3 and CRP) or developed an exacerbation (developed EXA; $n=34$ for Galectin-3, $n=33$ for CRP) during the course of the study. The data is presented as individual dots and median (bold line). Statistical analysis was performed using (A) a Kruskal-Wallis test followed by Dunn's multiple comparison test or (B-C) a Mann-Whitney $U$ test. 

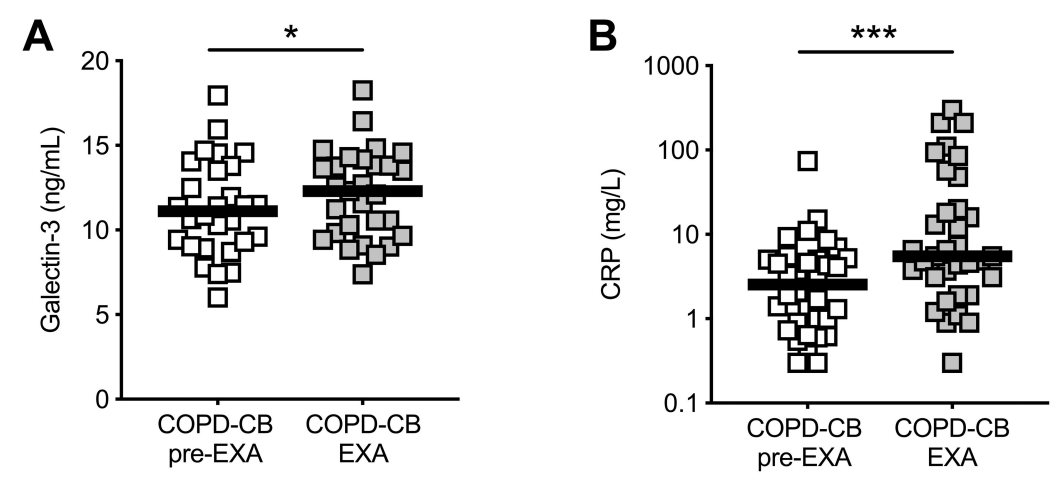

Figure 2 Serum levels of Galectin-3 during exacerbations. The levels of $(\mathbf{A})$ Galectin-3 $(n=29)$ and $(B)$ CRP $(n=34)$ were measured in serum within each patient with chronic obstructive pulmonary disease and chronic bronchitis (COPD-CB) during stable clinical conditions at the most recent visit prior to an exacerbation (pre-EXA; white squares) and during the exacerbation (EXA; grey squares). Certain patients suffered from an exacerbation within the first 15 weeks of the 15 months' course of the study, hence the inclusion sample constitute II of the 29 pre-EXA samples in (A) and 14 of the 34 pre-EXA samples in (B). The data is presented as individual dots and median (bold line). Statistical analysis was performed using Wilcoxon signed-rank test. Statistically significant differences are indicated as follows: $*_{p}$-value $<0.05$; $* * * p$-value $<0.00$ I.
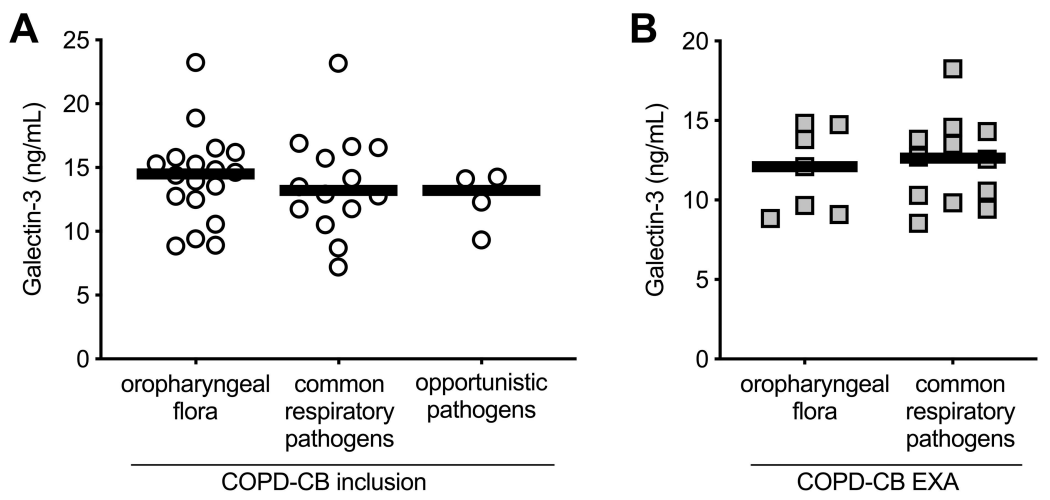

Figure 3 Serum levels of Galectin-3 in relation to bacterial growth in sputum samples. Sputum samples were obtained from patients with chronic obstructive pulmonary disease and chronic bronchitis (COPD-CB) during (A) stable clinical conditions at the time of inclusion $(n=36$, white dots) and $(B)$ and during exacerbation $(E X A$; $n=19$, grey squares) and the data show the level of Galectin-3 in serum in relation to the type of bacterial growth of oropharyngeal flora (inclusion, $n=18$, EXA $n=7$ ), common respiratory pathogens (inclusion, $n=14$, EXA $n=12$ ), and opportunistic pathogens (inclusion, $n=4$ ). The data is presented as individual dots and median (bold line). Statistical analysis was performed using (A), a Kruskal-Wallis test followed by Dunn's multiple comparison test or (B), a Mann-Whitney $U$ test.
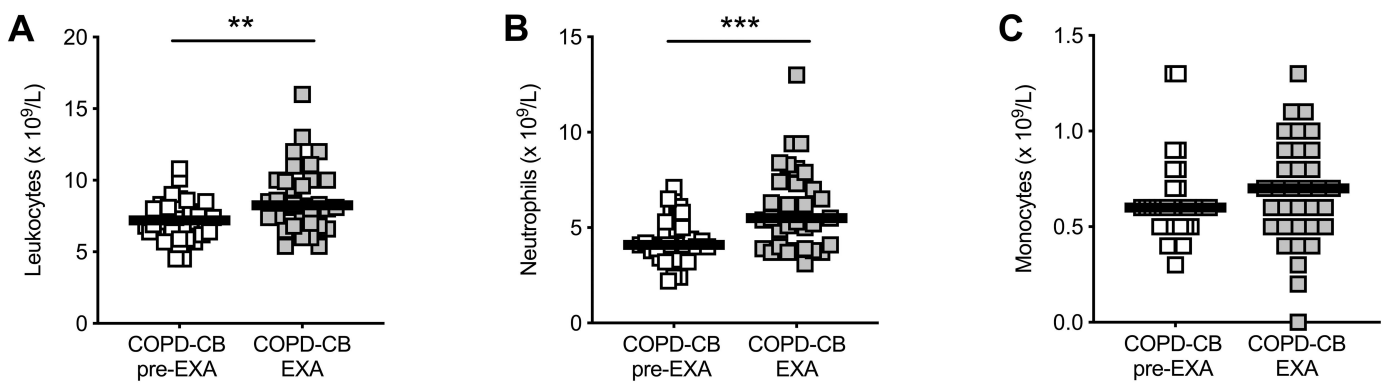

Figure 4 Alterations in total white blood cells and neutrophils during exacerbations. The absolute counts $\left(\times 10^{9} / \mathrm{L}\right)$ of $(\mathbf{A})$ leukocytes, $(\mathbf{B})$ neutrophils and $(\mathbf{C})$ monocytes were analyzed in blood from the same patients with chronic obstructive pulmonary disease and chronic bronchitis (COPD-CB) during stable clinical conditions at the most recent visit prior to an exacerbation (pre-EXA; $n=34$, white squares) and during the exacerbation (EXA; $n=34$, grey squares). Certain patients suffered from an exacerbation within the first 15 weeks of the 15 months' course of the study, hence the inclusion sample constitute 14 of the 34 pre-EXA samples in (A-C). The data is presented as individual dots and median (bold line). Statistical analysis was performed using Wilcoxon signed-rank test. Statistically significant differences are indicated as follows: $* *$-value $<0.01$; **** $p$-value $<0.001$. 


\section{Galectin-3 in Relation to CRP and Leukocytes During Exacerbations}

We observed no correlation between the levels of Galectin-3 and CRP during exacerbations, even though the level of both these proteins increased during these events (Figure 5A). We next examined if the increased level of Galectin-3 correlated with the increased level of total leukocytes, neutrophils or monocytes during exacerbations. However, no statistically significant correlation was detected (Figure 5B-D).

\section{Discussion}

In this longitudinal study, we characterized the level of Galectin-3 protein in serum samples from wellcharacterized patients with a history of COPD and CB and a confirmed status of current as well as historic smoking. Our results disclose that there is a reproducible but modest increase in the systemic levels of Galectin-3 during exacerbations. By optimizing the timing of sample collection during exacerbations, we avoided the confounding influence of emergency medication. Moreover, we investigated smokers with COPD-CB, being prone to exacerbations, ${ }^{35}$ and we ascertained that all of these patients were current smokers. In contrast, in the preceding study on systemic Galectin-3 by Feng et al patients with COPD were investigated without information about $\mathrm{CB}$, with inconsistent smoking status and uncertainties regarding sampling in relation to emergency medication. ${ }^{34}$ However, despite these differences in design, the study by Feng et al and our study show results along the same line, making it likely that systemic Galectin-3 is not very sensitive to common therapy against exacerbations.

We found that the average level of Galectin-3 in serum from the group of COPD-CB patients was indeed quite similar to the level in the HNS and LTS control group during stable clinical conditions. Indeed, our findings are
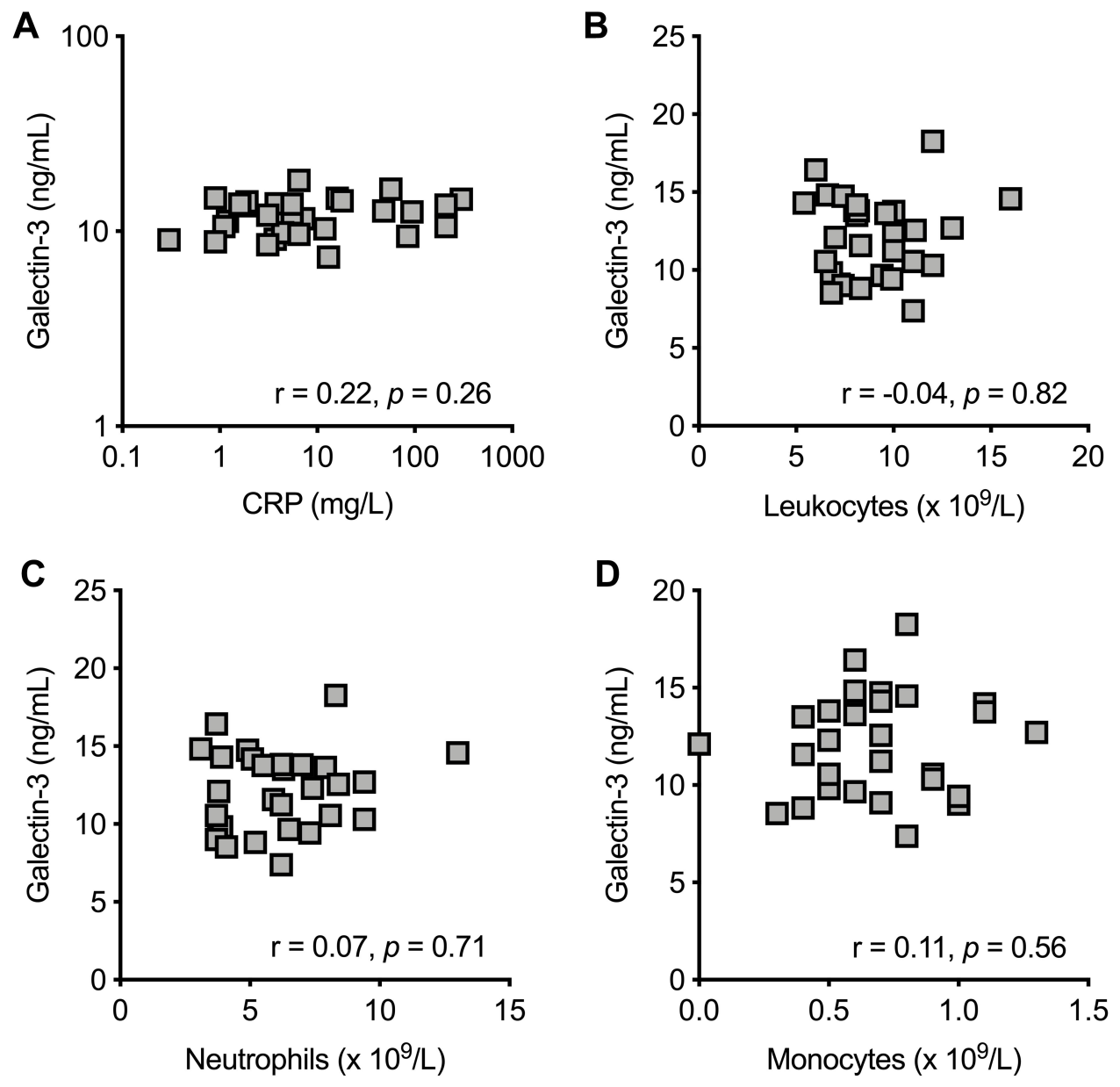

Figure 5 Galectin-3 versus CRP, white blood cells, neutrophils and monocytes during exacerbations. Correlations between the level of (A) CRP (measured in serum), and absolute counts of (B) leukocytes, (C) neutrophils and (D) monocytes (measured in blood) and the level of Galectin-3 (measured in serum) from patients with chronic obstructive pulmonary disease and chronic bronchitis (COPD-CB) during exacerbations $(n=29)$. Statistical analysis was performed using Spearman's rank correlation (the rho- and $p$-values are shown in each figure). 
corroborated by those in a previous study on plasma levels of Galectin-3 in patients with severe COPD without a diagnosis of $\mathrm{CB}$, and inconsistent smoking status, in comparison with healthy controls. ${ }^{44}$ Interestingly, a study on the local level of Galectin-3, assessed in bronchoalveolar lavage fluid, suggests a decrease in COPD patients compared to healthy controls during clinical stable conditions, suggesting a compartmental difference for systemic and local Galectin-3. ${ }^{45}$ Moreover, our current observations were made despite the detection of bacterial pathogens in certain patients with $\mathrm{COPD}$ and $\mathrm{CB}$; the presence of bacteria was not associated with increased Galectin-3 neither during exacerbations nor during stable clinical conditions. Tentatively, it seems as if local bacteria in the airways do not markedly alter systemic levels of Galectin-3 in smokers with COPD and CB.

We included measurements of CRP in serum as a reference biomarker in the current study, since this protein is regarded as a "golden standard" to assess systemic inflammation in patients with COPD. ${ }^{3,46}$ We found that CRP was clearly and reproducibly increased during exacerbations but this increase was modest; thereby confirming previous studies and suggesting a limited practical utility of CRP as a general biomarker for the exacerbation per se in the individual patient. In this respect, the scope of our current study is very different from that of the study from Butler et al, who examined and proved the utility of $\mathrm{CRP}$ as a tool for rational prescription of antibiotics to COPD patients with exacerbations. ${ }^{13}$ Because of the exacerbationrelated association of CRP and Galectin-3 that we observed, we investigated whether these two outcomes correlated but they did not. Possibly, this relates to that CRP and Galectin-3 have different cellular origins and release mechanisms. Along these lines, even though CRP may be synthesized by several cell types, the main bulk of CRP is produced by hepatocytes, ${ }^{47}$ whereas the main expression of Galectin-3 relates to myeloid cells. ${ }^{15,48}$ Such differences may explain why CRP displayed a consistent increase in smokers with COPD and CB even during stable clinical conditions ${ }^{37}$ whereas Galectin- 3 in the same group was similar to that in LTS and HNS.

We compared the level of Galectin-3 in serum during stable clinical conditions in patients with COPD and CB who developed exacerbations with those who did not. Notably, this comparison did not indicate any clear difference and the level of Galectin-3 in serum during stable clinical conditions did not correlate with the number of exacerbations. Thus, although the sample size was moderate and there is a certain risk for type II error, it is fair to state that our study failed to generate evidence in favor of
Galectin-3 possessing potential as a predictive biomarker for future exacerbations.

The included patients with COPD and CB had a record of historic exacerbations and displayed characteristic signs of systemic inflammation during exacerbations, beyond the enhanced CRP level. These signs included enhanced total levels of leukocytes including neutrophils, as previously reported. $^{37}$ Moreover, the included patients had a substantial tobacco load and the severity of COPD ranged from stage I to IV as defined by GOLD in $2011 .{ }^{38}$ Our current study material was larger than that investigated by Feng et al and, just like these authors, we excluded patients treated with oral or intravenous glucocorticoids, to avoid iatrogenic impact on immunological outcomes. Given these facts, we think that our study material is at least as relevant and representative as that studied by Feng et al. ${ }^{34}$

With reference to comorbidities other than chronic bronchitis, hypertension, osteoporosis, diabetes, hypothyroidism, sleep apnea, depression and anxiety, we interviewed the study subjects, checked their medical records, performed chest X-ray and tested lung function to exclude lung disease other than COPD-CB. With this approach, we also excluded severe congestive heart failure, ischemic heart disease, chronic inflammatory disease and malignancies. In a similar manner, Feng et al interviewed the study subjects, checked their medical records, performed chest $\mathrm{X}$-ray and tested lung function to exclude lung disease other than COPD-CB as well as ischemic heart disease, diabetes, autoimmune disease, chronic kidney disease and malignancies. ${ }^{34}$ Thus, in terms of the potentially confounding influence of comorbidities, this influence is likely to be modest in our study and in that by Feng et al. ${ }^{34}$

The actual levels (concentrations) of Galectin-3 protein detected in our current study cannot strictly be compared with those reported by Feng et al. ${ }^{34}$ This is because the referred authors utilized an ELISA to quantify Galectin-3 that was different from the one we utilized. However, it is interesting to note that the average increase during exacerbations was of a similar magnitude in both studies $(\sim 10 \%$ of the level during stable clinical conditions). Notably, the ELISA that we utilized has previously been used to quantify the level of Galectin-3 in a population of healthy humans encompassing more than one thousand subjects. ${ }^{49,50}$ The median level was then $10.9 \mathrm{ng} / \mathrm{mL}$ (interquartile range [IQR] 9.0-13.1 ng/ $\mathrm{mL})^{50}$ whereas it was $13.5 \mathrm{ng} / \mathrm{mL}$ (IQR $10.9-15.7 \mathrm{ng} / \mathrm{mL}$ ) during stable clinical conditions in our current study. In other words, the levels of Galectin-3 in the COPD-CB patients that we investigated are quite similar to those previously detected 
in healthy subjects, presumably because patients with severe comorbidities were excluded. ${ }^{49,50}$

\section{Conclusions}

Our longitudinal study on long-term smokers with a history of COPD and $\mathrm{CB}$ demonstrates a reproducible increase in the level of Galectin-3 in serum during exacerbations. However, the modest magnitude of this increase makes it less likely that systemic Galectin-3 will be clinically useful as a biomarker for exacerbations in this setting. Moreover, our study failed to provide evidence that Galectin-3 in serum distinguishes the patients who are prone to exacerbations from those who are not. Furthermore, our study indicates that the level of Galectin3 in serum during stable clinical conditions is quite similar to that in LTS or HNS, suggesting that systemic Galectin-3 is not pathogenically involved under these conditions in patients with COPD-CB. Although these results argue against Galectin- 3 as a useful biomarker in these patients, the potential clinical utility of targeting systemic Galectin-3 for detecting cardiovascular comorbidities remains to be addressed in this setting.

\section{Abbreviations}

$\mathrm{CB}$, chronic bronchitis; $\mathrm{COPD}$, chronic obstructive pulmonary disease; COPD-CB, chronic obstructive pulmonary disease and chronic bronchitis; CRP, C-reactive protein; DLCO, gas diffusion capacity for carbon monoxide; $\mathrm{FEV}_{1}$, forced expiratory volume in one second; FVC, forced vital capacity; GOLD, Global initiative for Chronic Obstructive Lung Disease; IQR, interquartile range; LTS, long-term smokers without COPD; HNS, healthy never-smokers.

\section{Ethics Approval and Informed Consent}

This study was conducted in accordance with the World Medical Association (the declaration of Helsinki) and approved by the regional ethics committee for medical research at the University of Gothenburg (S 233-03, T 28604 and $\mathrm{T}$ 521-06). Thus, all participants were included after oral and written informed consent.

\section{Acknowledgments}

The highly professional monitoring of study subjects and sample collection by Barbro Balder, B.Sc., at the Department of Respiratory Medicine and Allergology, Sahlgrenska University Hospital, is gratefully acknowledged posthumously.

\section{Author Contributions}

All authors contributed to data analysis, drafting or revising the article, have agreed on the journal to which the article was submitted, gave final approval of the version to be published, and agree to be accountable for all aspects of the work.

\section{Funding}

The study was supported by project funding from the Magnus Bergwall foundation (MS), the Rådman and Mrs Ernst Colliander foundation (MS), the Rune and Ulla Almlövs Foundation (MS), IFs foundation and Elisabeth and Alfred Ahlqvist's foundation - Swedish pharmacy Society (MS), the Swedish Medical Research Council (JB, AK and AL), federal funding under the ALF agreement for Region Västra Götaland (AK and $\mathrm{AL}$ ), federal funding under the ALF agreement for Region Stockholm (AL), the Arne and Inga-Britt Lundberg foundation (AK), the Swedish Heart-Lung Foundation (JB, AK and AL), the King Gustaf V 80years foundation (AK), the King Gustaf V's and Queen Victoria's Freemason Research Foundation (AL) and by federal funding from Karolinska Institutet (AL). The study sponsors were not involved in study design, data collection and analysis, decision to publish, or preparation of the manuscript. No funding was obtained from the tobacco industry.

\section{Disclosure}

The authors declare no financial or non-financial conflicts of interest for this work.

\section{References}

1. Lozano R, Naghavi M, Foreman K, et al. Global and regional mortality from 235 causes of death for 20 age groups in 1990 and 2010: a systematic analysis for the Global Burden of Disease Study 2010. Lancet. 2012;380(9859):2095-2128. doi:10.1016/S0140-6736(12) 61728-0

2. Global Strategy for the Diagnosis, Management and Prevention of COPD. Global Initiative for Chronic Obstructive Lung Disease (GOLD), 2020. Available from: www.goldcopd.org.

3. Global Strategy for the Diagnosis, Management and Prevention of COPD. Global Initiative for Chronic Obstructive Lung Disease (GOLD), 2019. Available from: www.goldcopd.org.

4. Li J, Sun S, Tang R, et al. Major air pollutants and risk of COPD exacerbations: a systematic review and meta-analysis. Int $J$ Chron Obstruct Pulmon Dis. 2016;11:3079-3091. doi:10.2147/COPD. $\mathrm{S} 122282$

5. Leuzzi G, Galeone C, Taverna F, Suatoni P, Morelli D, Pastorino U. C-reactive protein level predicts mortality in COPD: a systematic review and meta-analysis. Eur Respir Rev. 2017;26(143):160070. doi:10.1183/16000617.0070-2016 
6. Miller BE, Tal-Singer R, Rennard SI, et al. Plasma fibrinogen qualification as a drug development tool in chronic obstructive pulmonary disease. Perspective of the chronic obstructive pulmonary disease biomarker qualification consortium. Am J Respir Crit Care Med. 2016;193(6):607-613. doi:10.1164/rccm.201509-1722PP

7. MacNee W. Systemic inflammatory biomarkers and co-morbidities of chronic obstructive pulmonary disease. Ann Med. 2013;45 (3):291-300. doi:10.3109/07853890.2012.732703

8. Bchir S, Ben Nasr H, Garrouch A, et al. MMP-3 (-1171 5A/6A; Lys45Glu) variants affect serum levels of matrix metalloproteinase (MMP)-3 and correlate with severity of COPD: a study of MMP-3, MMP-7 and MMP-12 in a Tunisian population. J Gene Med. 2018;20 (1):e2999. doi:10.1002/jgm.2999

9. Linder R, Ronmark E, Pourazar J, Behndig A, Blomberg A, Lindberg A. Serum metalloproteinase-9 is related to COPD severity and symptoms - cross-sectional data from a population based cohort-study. Respir Res. 2015;16:28. doi:10.1186/s12931-0150188-4

10. Hawkins NM, Virani S, Ceconi C. Heart failure and chronic obstructive pulmonary disease: the challenges facing physicians and health services. Eur Heart J. 2013;34(36):2795-2803. doi:10.1093/eurheartj/eht192

11. Ho J, He W, Chan MTV, et al. Eosinophilia and clinical outcome of chronic obstructive pulmonary disease: a meta-analysis. Sci Rep. 2017;7(1):13451. doi:10.1038/s41598-017-13745-x

12. Brusselle G, Pavord ID, Landis S, et al. Blood eosinophil levels as a biomarker in COPD. Respir Med. 2018;138:21-31. doi:10.1016/j. rmed.2018.03.016

13. Butler CC, Gillespie D, White P, et al. C-reactive protein testing to guide antibiotic prescribing for COPD exacerbations. $N$ Engl J Med. 2019;381(2):111-120. doi:10.1056/NEJMoa1803185

14. Hughes RC. Mac-2: a versatile galactose-binding protein of mammalian tissues. Glycobiology. 1994;4(1):5-12. doi:10.1093/glycob/4.1.5

15. Diaz-Alvarez L, Ortega E. The many roles of galectin-3, a multifaceted molecule, in innate immune responses against pathogens. Mediators Inflamm. 2017;2017:9247574. doi:10.1155/ 2017/9247574

16. Johannes L, Jacob R, Leffler H. Galectins at a glance. J Cell Sci. 2018;131(9):jcs208884. doi:10.1242/jcs.208884

17. Liu FT, Hsu DK, Zuberi RI, Kuwabara I, Chi EY, Henderson WR Jr. Expression and function of galectin-3, a beta-galactoside-binding lectin, in human monocytes and macrophages. Am J Pathol. 1995;147(4):1016-1028.

18. Rovina N, Koutsoukou A, Koulouris NG. Inflammation and immune response in COPD: where do we stand? Mediators Inflamm. 2013;2013:413735. doi:10.1155/2013/413735

19. Di Stefano A, Capelli A, Lusuardi M, et al. Severity of airflow limitation is associated with severity of airway inflammation in smokers. Am J Respir Crit Care Med. 1998;158(4):1277-1285. doi:10.1164/ajrccm.158.4.9802078

20. Almkvist J, Karlsson A. Galectins as inflammatory mediators. Glycoconj J. 2002;19(7-9):575-581. doi:10.1023/B: GLYC.0000014088.21242.e0

21. Inohara H, Raz A. Functional evidence that cell surface galectin-3 mediates homotypic cell adhesion. Cancer Res. 1995;55 (15):3267-3271.

22. Kuwabara I, Liu FT. Galectin-3 promotes adhesion of human neutrophils to laminin. J Immunol. 1996;156(10):3939-3944.

23. Sato S, Ouellet N, Pelletier I, Simard M, Rancourt A, Bergeron MG. Role of galectin-3 as an adhesion molecule for neutrophil extravasation during streptococcal pneumonia. $J$ Immunol. 2002;168 (4):1813-1822. doi:10.4049/jimmunol.168.4.1813

24. Yamaoka A, Kuwabara I, Frigeri LG, Liu FT. A human lectin, galectin-3 (epsilon bp/Mac-2), stimulates superoxide production by neutrophils. J Immunol. 1995;154(7):3479-3487.
25. Karlsson A, Follin P, Leffler H, Dahlgren C. Galectin-3 activates the NADPH-oxidase in exudated but not peripheral blood neutrophils. Blood. 1998;91(9):3430-3438. doi:10.1182/blood. V91.9.3430

26. Karlsson A, Christenson K, Matlak M, et al. Galectin-3 functions as an opsonin and enhances the macrophage clearance of apoptotic neutrophils. Glycobiology. 2009;19(1):16-20. doi:10.1093/glycob/cwn104

27. Linden JR, Kunkel D, Laforce-Nesbitt SS, Bliss JM. The role of galectin-3 in phagocytosis of Candida albicans and Candida parapsilosis by human neutrophils. Cell Microbiol. 2013;15(7):1127-1142. doi:10.1111/cmi.12103

28. Iurisci I, Tinari N, Natoli C, Angelucci D, Cianchetti E, Iacobelli S. Concentrations of galectin-3 in the sera of normal controls and cancer patients. Clin Cancer Res. 2000;6(4):1389-1393.

29. Takenaka Y, Fukumori T, Raz A. Galectin-3 and metastasis. Glycoconj J. 2002;19(7-9):543-549. doi:10.1023/B: GLYC.0000014084.01324.15

30. Yu LG. Circulating galectin-3 in the bloodstream: an emerging promoter of cancer metastasis. World J Gastrointest Oncol. 2010;2 (4):177-180. doi:10.4251/wjgo.v2.i4.177

31. van der Velde AR, Meijers WC, Ho JE, et al. Serial galectin-3 and future cardiovascular disease in the general population. Heart. 2016;102(14):1134-1141. doi:10.1136/heartjnl-2015-308975

32. de Boer RA, Lok DJ, Jaarsma T, et al. Predictive value of plasma galectin-3 levels in heart failure with reduced and preserved ejection fraction. Ann Med. 2011;43(1):60-68. doi:10.3109/07853890. 2010.538080

33. Pilette C, Colinet B, Kiss R, et al. Increased galectin-3 expression and intra-epithelial neutrophils in small airways in severe COPD. Eur Respir J. 2007;29(5):914-922. doi:10.1183/09031936. 00073005

34. Feng W, Wu X, Li S, et al. Association of serum galectin-3 with the acute exacerbation of chronic obstructive pulmonary disease. Med Sci Monit. 2017;23:4612-4618. doi:10.12659/MSM.903472

35. Kim V, Han MK, Vance GB, et al. The chronic bronchitic phenotype of COPD: an analysis of the COPDGene Study. Chest. 2011;140 (3):626-633. doi:10.1378/chest.10-2948

36. Andelid K, Andersson A, Yoshihara S, et al. Systemic signs of neutrophil mobilization during clinically stable periods and during exacerbations in smokers with obstructive pulmonary disease. Int J Chron Obstruct Pulmon Dis. 2015;10:1253-1263. doi:10.2147/COPD.S77274

37. Andelid K, Tengvall S, Andersson A, et al. Systemic cytokine signaling via IL-17 in smokers with obstructive pulmonary disease: a link to bacterial colonization? Int J Chron Obstruct Pulmon Dis. 2015;10:689-702. doi:10.2147/COPD.S76273

38. Global Strategy for the Diagnosis, Management and Prevention of COPD. Global Initiative for Chronic Obstructive Lung Disease (GOLD); 2011. Available from: www.goldcopd.org.

39. Kim V, Criner GJ. Chronic bronchitis and chronic obstructive pulmonary disease. Am J Respir Crit Care Med. 2013;187(3):228-237. doi:10.1164/rccm.201210-1843CI

40. Cavailles A, Brinchault-Rabin G, Dixmier A, et al. Comorbidities of COPD. Eur Respir Rev. 2013;22(130):454-475. doi:10.1183/ 09059180.00008612

41. Ambade VN, Sontakke AN, Barthwal MS, Tyagi R, Basannar DR. Diagnostic utility of biomarkers in COPD. Respir Care. 2015;60 (12):1729-1742. doi:10.4187/respcare.03753

42. Dahl M, Vestbo J, Lange P, Bojesen SE, Tybjaerg-Hansen A, Nordestgaard BG. C-reactive protein as a predictor of prognosis in chronic obstructive pulmonary disease. Am J Respir Crit Care Med. 2007;175(3):250-255. doi:10.1164/rccm.200605-713OC

43. Deng ZC, Zhao P, Cao C, et al. C-reactive protein as a prognostic marker in chronic obstructive pulmonary disease. Exp Ther Med. 2014;7(2):443-446. doi:10.3892/etm.2013.1441 
44. Mueller T, Leitner I, Egger M, Haltmayer M, Dieplinger B. Association of the biomarkers soluble ST2, galectin-3 and growth-differentiation factor-15 with heart failure and other non-cardiac diseases. Clin Chim Acta. 2015;445:155-160. doi:10.1016/j.cca.2015.03.033

45. Mukaro VR, Bylund J, Hodge G, et al. Lectins offer new perspectives in the development of macrophage-targeted therapies for COPD/ emphysema. PLoS One. 2013;8(2):e56147. doi:10.1371/journal. pone. 0056147

46. Koutsokera A, Kiropoulos TS, Nikoulis DJ, et al. Clinical, functional and biochemical changes during recovery from COPD exacerbations. Respir Med. 2009;103(6):919-926. doi:10.1016/j.rmed.2008.12.006

47. Sproston NR, Ashworth JJ. Role of C-reactive protein at sites of inflammation and infection. Front Immunol. 2018;9:754. doi:10.3389/ fimmu.2018.00754
48. Sciacchitano S, Lavra L, Morgante A, et al. Galectin-3: one molecule for an alphabet of diseases, from A to Z. Int J Mol Sci. 2018;19 (2):379. doi:10.3390/ijms 19020379

49. Christenson RH, Duh SH, Wu AH, et al. Multi-center determination of galectin-3 assay performance characteristics: anatomy of a novel assay for use in heart failure. Clin Biochem. 2010;43(7-8):683-690. doi:10.1016/j.clinbiochem.2010.02.001

50. de Boer RA, van Veldhuisen DJ, Gansevoort RT, et al. The fibrosis marker galectin-3 and outcome in the general population. J Intern Med. 2012;272(1):55-64. doi:10.1111/j.1365-2796.2011.02476.x

\section{Publish your work in this journal}

The International Journal of COPD is an international, peer-reviewed journal of therapeutics and pharmacology focusing on concise rapid reporting of clinical studies and reviews in COPD. Special focus is given to the pathophysiological processes underlying the disease, intervention programs, patient focused education, and self management protocols. This journal is indexed on PubMed Central, MedLine and CAS. The manuscript management system is completely online and includes a very quick and fair peer-review system, which is all easy to use. Visit http://www.dovepress.com/testimonials.php to read real quotes from published authors. 